\title{
A Case of Meningococcal and HSV-2 Meningitis in a Patient Being Treated with Ustekinumab for Pityriasis Rubra Pilaris
}

\author{
Charalampos I Kalogeropoulos, Konstantinos A Papathanasiou, Ismini Tsagkaraki, \\ Georgios Giannopoulos, Aristotelis Bamias, Eleni I Boutati
}

Second Propaedeutic Department of Internal Medicine and Research Institute, University General Hospital Attikon, National and Kapodistrian University of Athens, Athens, Greece

\section{Doi: 10.12890/2020_001615 - European Journal of Case Reports in Internal Medicine - ๔ EFIM 2020}

Received: 04/03/2020

Accepted: $11 / 03 / 2020$

Published: $22 / 05 / 2020$

How to cite this article: Kalogeropoulos CI, Papathanasiou KA, Tsagkaraki I, Giannopoulos G, Bamias, Boutati El. A case of meningococcal and HSV-2 meningitis in a patient being treated with ustekinumab for pityriasis rubra pilaris. EJCRIM 2020;7: doi:10.12890/2020_001615.

Conflicts of Interests: The Authors declare that there are no competing interests.

This article is licensed under a Commons Attribution Non-Commercial 4.0 License

HOSPITAL GRAND ROUNDS

University General Hospital Attikon, by Editorial Board Member Eleni Boutati

TThe University General Hospital «Attikon», Athens, Greece, is a tertiary University teaching hospital. It was founded in 2002 and first patients admitted in November 2003. Nowadays it has a capacity of 677 beds, with 22 University Clinics, and with over 2500 employees and 500 staff physicians representing more than 60 specialties and subspecialties. It is the only General University Hospital in Athens. The hospital is managed by the Health National System of Greece and all Clinics, Units and Laboratories are coordinated by the School of Medicine of the National and Kapodistrian University of Athens. Its main mission is to provide secondary and tertiary care to the citizens, to support the operation of University Clinics and Special Units, to train medical students, doctors, and other health care professionals, as well as to promote research development in the health sector.

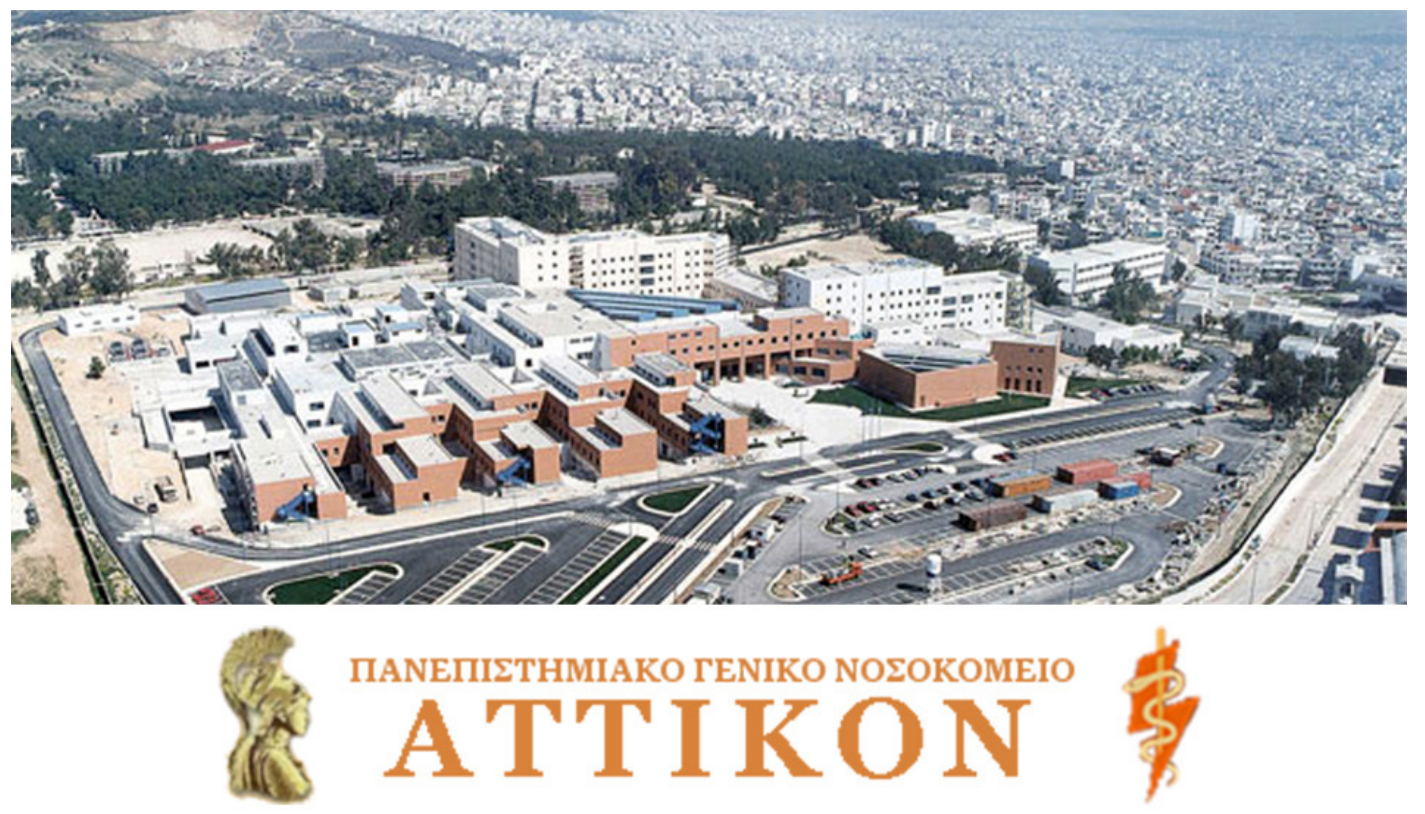




\section{ABSTRACT}

Pityriasis rubra pilaris (PRP) is a rare chronic inflammatory papulosquamous dermatosis affecting both adults and children. Six subtypes of PRP have been described. Recently, the management of PRP with biologic immunosuppressive agents regularly used in psoriasis has been supported by several case reports and series. Ustekinumab is an anti-IL12/23 IgG1 kappa human monoclonal antibody. It has been approved for the treatment of Crohn's disease, plaque psoriasis, psoriatic arthritis and ulcerative colitis. It has also been reported to be effective as an off-label treatment for PRP. Current data are equivocal regarding infectious disease risk with ustekinumab administration. We describe a case of meningococcal and HSV-2 infection of the central nervous system in a patient being treated with ustekinumab for PRP.

\section{LEARNING POINTS}

- The administration of biologic immunosuppressive agents can result in severe life-threatening infections.

- Research is required on the infection potential of ustekinumab.

- Physicians should be aware of the possibility of infectious disease when prescribing biologic agents.

- Vaccination is essential in immunosuppressed adults.

\section{KEYWORDS}

Pityriasis rubra pilaris (PRP), ustekinumab, meningococcal meningitis

\section{INTRODUCTION}

Pityriasis rubra pilaris (PRP) refers to a group of rare skin conditions found in both adults and children and characterized by chronic inflammatory papulosquamous dermatosis. Six subtypes of PRP have been described. Follicular palmoplantar hyperkeratosis and peachy coral plaques affecting the head, trunk and limbs are common to all subtypes. PRP can occasionally progress to a generalized erythroderma, with 'islands of sparing', which are areas of unaffected skin considered the hallmark of this disease. Its aetiopathogenesis is still uncertain, but current evidence suggests a multifactorial origin. The vast majority of cases are sporadic, although familial autosomal dominant and recessive patterns of inheritance have been suggested ${ }^{[1]}$. Treating PRP is a clinical challenge since some cases are self-limiting and do not require treatment, while others are unresponsive to any treatment combination. This management complexity together with the disease's rarity may partly explain the lack of standard treatment guidelines. Most dermatologists support combination therapies with administration of systemic agents and topical therapy. Topical agents for symptomatic treatment include emollients, keratolytic agents (such as urea), topical corticosteroids, tazarotene and topical calcineurin inhibitors. Oral retinoids are the first-line option for systemic treatment. Methotrexate and UV-light therapy (phototherapy) in combination with retinoids, as well as cyclosporin A have also been used. However, the risk of adverse effects with combination therapy is higher than with monotherapy. Recently, several case reports and series have supported the use of biologic immunosuppressive agents, including TNF-alpha inhibitors (infliximab, etanercept) as well as ustekinumab and secukinumab, in PRP patients unresponsive to conventional treatment ${ }^{[1,2]}$.

Ustekinumab is an anti-IL12/23 IgG1 kappa human monoclonal antibody. It has been approved for the treatment of moderately to severely active Crohn's disease, plaque psoriasis, psoriatic arthritis, and recently ulcerative colitis ${ }^{[3]}$. It has been reported to be effective in PRP as an off-label treatment given the fact that treatment options are limited ${ }^{[4-7]}$. Common side effects of ustekinumab include injection site reactions, flu-like symptoms, headache, fatigue, diarrhoea, skin rash and itching ${ }^{[3]}$. It has been postulated that, as a biologic treatment, it can increase the chance of serious infections (including tuberculosis, and bacterial, viral and fungal infections), skin cancer, reversible posterior leukoencephalopathy syndrome (RPLS) and hypersensitivity reactions. An analysis of 2014 PSOLAR data (an observational study of 12,093 patients with psoriasis) identified no increased risk of malignancy, major adverse cardiovascular events, serious infection or mortality with ustekinumab ${ }^{[8]}$.

We report a case of meningococcal and HSV-2 infection of the central nervous system (CNS) in a patient being treated with ustekinumab for PRP. To the best of our knowledge, there are only two other reported cases of CNS infection in patients treated with ustekinumab ${ }^{[9,10]}$.

\section{CASE DESCRIPTION}

An intubated 60-year-old man was urgently transferred by air from a rural hospital to our emergency department as a possible case of CNS infection necessitating intensive care treatment in a higher-level tertiary hospital. The patient's history showed acute onset of fever up to $38.5^{\circ} \mathrm{C}$ accompanied by rigor, neck stiffness, vomiting and confusion lasting 3 days. He had initially presented to the emergency department of a rural hospital, where he underwent basic laboratory evaluation. An emergent computed tomography (CT) brain scan did not demonstrate any abnormal findings. The patient was considered for a lumbar puncture due to marked nuchal rigidity. Since the blood tests revealed a mild increase in INR and the rural hospital lacked neurosurgical facilities, cerebrospinal fluid (CSF) examination was temporarily postponed and the patient was administered an intravenous dose of ceftriaxone within the first hour of medical contact. The patient's mental status 
rapidly deteriorated and it was decided to intubate him for airway protection. The patient was then admitted to our hospital a few hours after intubation.The patient had a 10-year history of PRP, a bilateral saphenectomy 10 years previously for venous insufficiency of the lower extremities, and frequent episodes of herpes simplex virus infections (herpes labialis) since childhood. He quit smoking several years ago and drinks alcohol only on rare social occasions. The patient's PRP was initially managed with acitretin and topical steroids. After 6 years of treatment and because of repeated skin lesion relapses, the patient's dermatologist decided to escalate treatment with the addition of subcutaneous injections of ustekinumab $45 \mathrm{mg}$ at week 0 and week 4 and every 12 weeks thereafter. The patient had regular medical checkups every 6 months, with no abnormal findings in the most recent laboratory work-up (Table 1) 2 months previously.

Additionally, pre-treatment tests for latent mycobacterium tuberculosis infection, viral hepatitis (HBV, HCV) and human immunodeficiency virus (HIV) were all negative. The patient had received his last injection of ustekinumab 40 days previously and was not on any additional medication.

\begin{tabular}{|c|c|c|}
\hline Parameter (blood) & Results & Normal range \\
\hline Leucocytes $(K / \mu l)$ & 7.62 & $4.00-10.00$ \\
\hline Platelets (K/ $\mu \mathrm{l})$ & 188 & $150.0-400.0$ \\
\hline Haemoglobin (g/dl) & 13.5 & $13.5-18.0$ \\
\hline Haematocrit (\%) & 40.8 & $40.0-54.0$ \\
\hline$C R P(m g / d l)$ & 0.1 & $<0.5$ \\
\hline Creatinine (mg/dl) & 0.8 & $0.6-1.3$ \\
\hline $\operatorname{Urea}(\mathrm{mg} / \mathrm{dl})$ & 34 & $10-50$ \\
\hline SGOT (U/I) & 18 & $<37$ \\
\hline SGPT (U/I) & 16 & $<41$ \\
\hline$\gamma-G T(U / I)$ & 16 & $0-16$ \\
\hline$A L P(U / I)$ & 70 & $40-129$ \\
\hline $\mathrm{LDH}(\mathrm{U} / \mathrm{I})$ & 164 & $135-225$ \\
\hline Anti-HIVI, II & Negative & Negative \\
\hline$H B s A g(I U)$ & Negative, 0.4 & Negative $<0.9$ \\
\hline Anti-HCV (IU) & Negative, 0.1 & Negative $<0.9$ \\
\hline
\end{tabular}

Table 1. Normal blood test results 20 days before the patient's last ustekinumab injection

At presentation to our hospital, the patient was intubated, under mechanical ventilation and haemodynamically stable. The clinical examination revealed mild nuchal rigidity, with no rash or signs of increased intracranial pressure. His pupils were of equal and normal size, and reactive to light. The rest of the examination was unremarkable. A repeat CT of the brain showed no changes compared with the baseline imaging obtained a few hours previously. Due to high clinical suspicion of CNS infection and apparent immunosuppression, the patient was given empiric broad-spectrum antibiotics, adding intravenous ampicillin/sulbactam, vancomycin and acyclovir to ceftriaxone. The admission electrocardiogram showed a normal sinus rhythm with 1:1 atrioventricular conduction, while a supine chest x-ray showed the tracheal tube in the correct place with no signs of lung infiltrates or cardiac silhouette abnormalities. The admission arterial blood gas analysis (on $\mathrm{FiO}_{2}$ $100 \%$, respiratory rate $16 / \mathrm{min}$, tidal volume $500 \mathrm{ml}$ and positive end-expiratory pressure $5 \mathrm{~cm} \mathrm{H}_{2} \mathrm{O}$ ) showed pH $7.42, \mathrm{pCO}_{2} 40.6 \mathrm{mmHg}_{2} \mathrm{pO}_{2}$ $350 \mathrm{mmHg}, \mathrm{HCO}_{3} 26 \mathrm{mmol} / \mathrm{l}$, lactic acid $1.2 \mathrm{mmol} / \mathrm{l}$, and SO $100 \%$. As part of our hospital protocol, blood tests were repeated and revealed an increase in white blood cell count and in C-reactive protein (CRP), as well as a normocytic normochromic anaemia and thrombocytopenia. INR was 0.96 (Table 2). The patient was transferred to our hospital's intensive care unit (ICU), where a lumbar puncture was performed. CSF examination revealed $5.440 / \mathrm{mm}^{3}$ cells with polymorphonuclear predominance, low glucose $18 \mathrm{mg} / \mathrm{dl}$ (blood glucose was $120 \mathrm{mg} / \mathrm{dl}$ ) and high protein levels (477 mg/dl) (Table 3). Gram stain revealed gram-negative diplococci, while PCR examination identified both Neisseria meningitidis serogroup B and HSV-2. During his stay in the ICU, the patient presented persistent euvolemic hyponatremia that was attributed to inappropriate secretion of antidiuretic hormone (SIADH) due to the CNS infection. 


\section{of Case Reports in \\ Internal Medicine}

\begin{tabular}{|c|c|c|}
\hline Parameter (blood) & Results & Normal range \\
\hline Leucocytes (K/Ml) & 11.14 & $4.00-11.00$ \\
\hline Neutrophils (\%) & 86.9 & $40.0-75.0$ \\
\hline Platelets (K/Ml) & 75 & $150.0-400.0$ \\
\hline Haemoglobin (g/dl) & 11.0 & $13.5-17.5$ \\
\hline Haematocrit (\%) & 31.4 & $41.0-53.0$ \\
\hline$M C V(f f)$ & 84.9 & $76.0-96.0$ \\
\hline $\mathrm{MCHC}(\mathrm{g} / \mathrm{dl})$ & 35.0 & $30.0-36.0$ \\
\hline$C R P(m g / d l)$ & 441.0 & $0-6.0$ \\
\hline Creatinine ( $\mathrm{mg} / \mathrm{dl})$ & 0.5 & $0.7-1.2$ \\
\hline Urea (mg/dl) & 46.6 & $16.6-48.5$ \\
\hline AST/SGOT (U/I) & 14 & $<40$ \\
\hline ALT/SGPT (U/I) & 13 & $<41$ \\
\hline$\gamma$-GT (U/I) & 24 & $8.0-61.0$ \\
\hline$A L P(U / I)$ & 53 & $40-129$ \\
\hline PT (sec) & 10.86 & $10.0-13.00$ \\
\hline INR & 0.96 & $0.90-1.20$ \\
\hline APTT (sec) & 34.82 & $24.00-39.00$ \\
\hline Fibrogen (mg/dl) & 988.5 & $200.0-400.0$ \\
\hline $\mathrm{Na}(\mathrm{mmol} / \mathrm{l})$ & 135 & $136-146$ \\
\hline$K(\mathrm{mmol} / \mathrm{l})$ & 4.1 & $3.5-5.1$ \\
\hline $\mathrm{Ca}(\mathrm{mg} / \mathrm{dl})$ & 8.3 & $8.6-10.3$ \\
\hline $\mathrm{LDH}(\mathrm{U} / \mathrm{I})$ & 411 & $135-225$ \\
\hline$C K(U / I)$ & 102 & $39-308$ \\
\hline Troponin (pg/ml) & 8.4 & $<14$ \\
\hline
\end{tabular}

\begin{tabular}{|l|l|}
\hline Parameter (CSF) & Results \\
\hline Number of cells (/mm $)^{3}$ & 5.440 \\
\hline Polymorphonuclear neutrophils (\%) & 90 \\
\hline Number of RBC (/mm $)^{3}$ & 960 \\
\hline Colour & Light yellow \\
\hline Appearance & Slightly cloudy \\
\hline Total protein (mg/dl) & 477.0 \\
\hline Albumin (mg/dl) & 138.20 \\
\hline Glucose (mg/dl) & 18 \\
\hline LDH (U/l) & 95 \\
\hline Gram strain & Gram-negative diplococcus \\
\hline PCR (film array) & $\begin{array}{l}\text { Neisseria meningitidis serogroup B(+) } \\
\text { Herpes simplex virus 2 (+) }\end{array}$ \\
\hline
\end{tabular}

Table 2. Blood test results in our emergency department

Table 3. CSF examination results

The patient progressively improved over the following days, displaying normalization of inflammatory markers and hyponatremia. After 5 days of intensive care treatment, the patient was successfully weaned from the endotracheal tube and transferred to our internal medicine ward for further management. At that point, he was haemodynamically stable and afebrile. Apart from slight confusion, the patient's progress was initially uncomplicated while he was on ceftriaxone and acyclovir. However, on day 4 of his stay in our ward, he started to exhibit spiking fevers up to $38.5^{\circ} \mathrm{C}$. Over the following 3 days he displayed abnormally elevating liver function tests (transferase enzymes) in combination with persistent fever but no new signs or symptoms. After infectious specialist team consultation and negative blood and urine cultures, we decided to stop intravenous antibiotics. Two days later, the patient became afebrile and his liver function tests returned to normal, suggesting the fever was caused by drugs. Thorough imaging analysis was requested (CT of the brain, chest and abdomen) but results were unremarkable. After 3 weeks of hospitalization, the patient was discharged ambulatory, afebrile and restored back to his previous excellent health. Our recommendation to his treating dermatologist was to halt ustekinumab for PRP. The patient was strongly advised to get vaccinated with pneumococcal (PCV-13, PPSV-23) and inactivated influenza vaccines as well as with meningococcal group B and groups A, C, W135 and Y vaccines. Follow-up in the patient's local hospital 1 month later showed an unremarkable clinical examination and normal blood tests.

\section{DISCUSSION}

N. meningitidis and herpes simplex type 2 are common causes of CNS infection in adults. N. meningitidis, a gram-negative diplococcus and obligate human pathogen, normally colonizes the nasopharynx and is typically found in $10 \%$ of asymptomatic carriers. In Europe and the 
USA, serogroup B is the main type causing meningococcal disease. Patients with meningococcal meningitis usually present with acute onset of severe headache, fever, nausea, vomiting, photophobia and a stiff neck as well as lethargy, altered mental status or prolonged fever, especially the elderly. Another common manifestation is a diffuse petechial rash especially on the trunk and lower extremities. Risk factors predisposing to meningococcal disease include congenital complement deficiencies or dysfunctional properdin, asplenia (anatomical or functional), HIV infection and smoking or concurrent viral infections of the upper respiratory tract ${ }^{[11]}$. Although it is thought ustekinumab may be implicated in some cases of serious infection ${ }^{[12]}$, there are still conflicting arguments concerning the potential of biologic agents like ustekinumab to increase this risk ${ }^{[13]}$. According to the European Society of Clinical Microbiology and Infectious Diseases (ESCMID) Study Group for Infections in Compromised Hosts (ESGICH) Consensus, ustekinumab should be considered to pose a low risk for infections, as most infections are limited to the upper respiratory tract and only a few cases of opportunistic infections or TB reactivation have been reported, as shown in reports from four psoriasis trials where patients were exposed to ustekinumab for up to 3 years ${ }^{[14]}$. Note that our patient had been exposed ustekinumab for 4 consecutive years.

There are only two other reported cases of ustekinumab associated with CNS infection ${ }^{[9,10]}$, and no reports of meningococcal meningitis or combined viral-bacterial meningitis in patients treated with this biologic agent.

The quality of evidence-based medicine presented in case reports may be low, but they can still teach us valuable everyday clinical practice lessons. Specifically, our previously healthy patient was exposed to an immunosuppressive agent associated with a low risk of infection but which eventually endangered his life by impairing the efficiency of his immune system. The patient may have had genetic predisposing factors, especially since genome-wide association studies have identified single nucleotide polymorphisms that render carriers prone to bacterial meningitis and cold sores, among other common infections ${ }^{[15]}$. We believe that, until the ability of novel biologic agents to trigger infections has been elucidated, clinicians should be vigilant when prescribing such medications. We also stress the importance of vaccination and strongly recommend the vaccination status of any patient starting on biologic agents should be meticulously revised well in advance of medication commencement so their immune system can be improved.

\section{REFERENCES}

1. Klein A, Landthaler M, Karrer S. Pityriasis rubra pilaris: a review of diagnosis and treatment. Am J Clin Dermatol 2010;11(3):157-170

2. Kromer C, Sabat R, Celis D, Mössner R. Systemic therapies of pityriasis rubra pilaris: a systematic review. J Dtsch Dermatol Ges 2019;17(3):243-259.

3. emc. STELARA $45 \mathrm{mg}$ and $90 \mathrm{mg}$, solution for injection (vials) and solution for injection in prefilled syringe. Summary of product characteristics. Available from: https://www. medicines.org.uk/emc/medicine/32569. Accessed 26 March 2020.

4. Wohlrab J, Kreft B. Treatment of pityriasis rubra pilaris with ustekinumab. Br J Dermatol 2010;163(3):655-656.

5. Chowdhary M, Davila U, Cohen DJ. Ustekinumab as an alternative treatment option for chronic pityriasis rubra pilaris. Case Rep Dermatol 2015;7(1):46-50.

6. Napolitano M, Lembo L, Fania L, Abeni D, Didona D, Didona B. Ustekinumab treatment of pityriasis rubra pilaris: a report of five cases. J Dermatol 2018;45(2):202-206.

7. Aragón-Miguel R, Prieto-Barrios M, Calleja-Algarra A, Velasco-Tamariz V, Andres-Lencin JJ, Ortiz-Romero P, et al. Refractory pityriasis rubra pilaris with good response after treatment with ustekinumab. J Dtsch Dermatol Ges 2018;16(8):1022-1025.

8. Papp K, Gottlieb AB, Naldi L, Pariser D, Ho V, Goyal K, et al. Safety surveillance for ustekinumab and other psoriasis treatments from the Psoriasis Longitudinal Assessment and Registry (PSOLAR). J Drugs Dermatol 2015;14(7):706-714.

9. Stöllberger C, Finsterer J. Varicella zoster virus meningitis under ustekinumab because of plaque psoriasis. J Dermatol 2017:44(6):703-705.

10. Van Den Tooren HK, Bharambe V, Silver N, Michael BD. Herpes simplex virus encephalitis in a patient receiving ustekinumab associated with extensive cerebral edema and brainshift successfully treated by immunosuppression with dexamethasone. BMJ Case Rep 2019;12(8).

11. Mount HR, Boyle SD. Aseptic and bacterial meningitis: evaluation, treatment, and prevention. Am Fam Physician 2017;96(5):314-322.

12. Janssen Biotech, Inc. STELARA® (ustekinumab). Highlights of prescribing information. Available from http://www.stelarahcp.com/pdf/Prescribinglnformation.pdf. Accessed 20 February 2020.

13. Sehgal VN, Pandhi D, Khurana A. Biologics in dermatology: adverse effects. Int J Dermatol 2015;54(12):1442-1460.

14. Winthrop KL, Mariette X, Silva JT, Benamu E, Calabrese LH, Dumusc A, et al. ESCMID Study Group for Infections in Compromised Hosts (ESGICH) Consensus Document on the safety of targeted and biological therapies: an infectious diseases perspective (Soluble immune effector molecules [II]: agents targeting interleukins, immunoglobulins and complement factors). Clin Microbiol Infect 2018;24:S21-40.

15. Tian C, Hromatka BS, Kiefer AK, Eriksson N, Noble SM, Tung JY, et al. Genome-wide association and HLA region fine-mapping studies identify susceptibility loci for multiple common infections. Nat Commun 2017;8(1):599. 